\title{
Effects of Zero Mode and Thin Spectrum on the Life Time of Atomic Bose Einstein Condensates
}

\author{
T. Birol \\ Department of Physics, Cornell University, Ithaca, 14853 NY, U.S.A. and \\ Department of Physics, Koç University, Sarvyer, 34450, Istanbul, Turkey \\ Ö. E. Müstecaplığlu \\ Department of Physics, Koç University, Sarryer, Istanbul, 34450, Turkey
}

\begin{abstract}
Reviewing the ideas developed in [1], the ground state life time of a finite size atomic Bose Einstein condensate is studied for coherent, squeezed coherent and thermal coherent ground states. Ground state evolution of coherent and squeezed coherent states in a double well potential is studied. Effects of thin spectrum on Bose-Einstein condensates is discussed and quasiparticle excitation lifetimes are calculated. It is shown that the effect of the states we use on the free energy vanishes in the thermodynamic limit. Possible extension to a double well potential and effect of a second broken symmetry is also discussed.
\end{abstract}

PACS numbers:

\section{INTRODUCTION}

Shortly after Bose Einstein condensation [2] was obtained in trapped Alkali atoms [3], many theoretical and experimental studies focused on the quantum coherence properties of such systems. It was shown that apart from the usual decoherence, which stems from the imperfect isolation from the environment, the system also suffers phase diffusion [4, 5], which is due to the atomic number fluctuations in the condensate [6]. There is a third source of decoherence which limits the life time of excitations in BECs. This mechanism is based on the existence of a group of thin spectrum states [7]. The relation of thin spectra with decoherence of excitations is recently proposed and applied to Lieb-Mattis model and superconductors by the same authors [8, [9]. Effect of a thin spectrum on quasiparticle excitations on BECs is discussed in [1]. In this paper we review these ideas with some extensions and also discuss the decoherence that a double well BEC experiences.

This paper is organized as follows: Section II begins with a review of a toy model for zero mode dynamics, studying coherent, squeezed coherent and thermal coherent ground state lifetimes. A toy model for a double well condensate is also introduced and coherent and squeezed coherent states in such a system is studied. In section III we apply the thin spectrum formalism to atomic BECs and discuss its similarities and differences with other calculations. After making some comments about the existence of a second thin spectrum in a double well system and outlining the calculation of lifetime in a double well system, we conclude in section IV. Acknowledgements are in section $\mathrm{V}$.

\section{TOY MODEL}

\section{A. Introduction}

In order to understand the basic idea underlying the phase diffusion at zero temperature, it is useful to introduce a toy model [10, 11]. The total Hamiltonian of a homogeneous Bose Einstein condensate in the weakly interacting limit is

$$
\mathcal{H}=\sum_{k} E_{k} a_{k}^{\dagger} a_{k}+\frac{\tilde{u}}{2} \sum_{k, p, q} a_{p+q}^{\dagger} a_{k-q}^{\dagger} a_{k} a_{p}
$$

where $a_{k}$ is the annihilation operator of the $k$ mode and $\tilde{u}$ is the parameter determining the strength of interactions between bosons, that is $\tilde{u}=\frac{4 \pi \hbar^{2} a_{s}}{m V}$, where $V$ is the quantization volume. In order to fix the average number of atoms, a chemical potential $\mu$ is also included in single particle energy: $E_{k}=\frac{\hbar^{2} k^{2}}{2 m}-\mu$.

We are interested in the zero-mode dynamics of the system, so we discard terms including $a_{\vec{k} \neq 0}$ and redefine $\mu$ in order to get the basic $U(1)$ gauge symmetric Hamiltonian:

$$
\mathcal{H}=\frac{\tilde{u}}{2} a^{\dagger} a^{\dagger} a a-\mu a^{\dagger} a,
$$

The grounstate of such a Hamiltonian is clearly a Fock state with a number $N$ determined by $\mu$. However, Fock states have no definite phase and since Bose Einstein condensation entails a phase-symmetry broken state, the groundstate we seek cannot simply be a Fock state. The simplest idea is to consider a coherent state $|\alpha\rangle$ with $\alpha=\sqrt{N}$, as coherent states are the simplest states carrying a (almost) definite phase. A second step can be considering squeezed coherent states [12], which again carry some phase information, but the uncertainty in their phase can be larger or less than a corresponding coherent state. And finally, it is important to find a state 
which both carries phase information and is temperature dependent. For this purpose, we are going to introduce the thermal coherent states after studying the zero-mode evolutions of coherent and squeezed coherent states.

\section{B. Lifetime for coherent groundstate}

The coherent (or quasi-classical [13]) state $|\alpha\rangle$ is defined as the right eigenstate of the annihilation operator and has the Fock state expansion

$$
|\alpha\rangle=\mathrm{e}^{-|\alpha|^{2} / 2} \sum_{n=0}^{\infty} \frac{\alpha^{n}}{\sqrt{n !}}|n\rangle .
$$

This simple expansion makes it possible to calculate the time dependence of the expectation value of the annihilation operator $a$, which we consider as the order parameter, possible. We define the energy of the $n$-th Fock state $|n\rangle$ as $E_{n}=\frac{\tilde{u}}{2}\left(n^{2}-n\right)-\mu n$ through $\mathcal{H}|n\rangle=E_{n}|n\rangle$. This gives the simple time dependent expression

$$
\langle\alpha|a| \alpha\rangle=\sqrt{N} \exp \left(N\left[\mathrm{e}^{-\frac{i}{\hbar} \tilde{u} t}-1\right]\right) \mathrm{e}^{\frac{i}{\hbar} \mu t},
$$

whose short time behavior is found to be

$$
\langle\alpha|a| \alpha\rangle=\sqrt{N} \mathrm{e}^{\frac{i}{\hbar} \mu t} \mathrm{e}^{-i \frac{N \tilde{u}}{\hbar} t} \mathrm{e}^{-\frac{N \tilde{u}^{2}}{2 \hbar^{2}} t^{2}} .
$$

Therefore, the order parameter decays exponentially [4, 5]. At longer time scale $\langle\alpha|a| \alpha\rangle$ revives due to discrete and thus periodic nature of the exact time evolution (44). However, the ratio of the revival time $t_{r}$ to the collapse time scales as $t_{r} / t_{c}=\sqrt{N}$, and hence in the thermodynamic limit the collapse is irreversible. Denoting the density of condensed atom numbers in the quantization volume as $\rho=N / V$, collapse time $t_{c}=\hbar / \sqrt{N} \tilde{u}$ can be written as $t_{c}=\hbar \sqrt{N} / \rho u_{0}$ to see its behavior in the thermodynamic limit more directly. As $\rho$ is fixed in the thermodynamic limit where $N$ and $V$ increases indefinitely, we see that $t_{c}$ increases indefinitely. Revival time $t_{r}=\hbar / \tilde{u}=\hbar N / u_{0} \rho$ increases with $N$ linearly. In practice, the available condensates contain small number of atoms and furthermore, they are in traps that makes them inhomogeneous systems. Our homogeneous system Hamiltonian can qualitatively describe their collapse time behavior by letting $V$ denote the condensate mode volume, though $t_{c}, t_{r}$ would have different expressions for the case of a trapped condensate. In particular, collapse time of a harmonically trapped three dimensional isotropic condensate in a coherent state behaves like $t_{c} \sim N^{1 / 10}$ in the Thomas-Fermi limit [1]. Homogenous condensate collapse time is growing much faster, as $t_{c} \sim N^{1 / 2}$. Despite these quantitative differences, we can still express $t_{c}$ of homogeneous BEC in terms of parameters of a trapped BEC. For that aim we shall only eliminate $m$ via the characteristic length scale for a harmonic trap potential as $a_{\mathrm{ho}}=\sqrt{\hbar / m \omega_{\mathrm{tr}}}$ in terms of the harmonic trap frequency $\omega_{\text {tr }}$. We find

$$
t_{c}=\frac{\sqrt{N}}{4 \pi N_{\mathrm{eff}}} \frac{1}{\omega_{\mathrm{tr}}},
$$

where $N_{\text {eff }}=\rho a_{\text {ho }}^{2} a_{s}$. Assuming a typical situation of current experiments with $N \sim 10^{6}, a_{s}=10 \mathrm{~nm}, a_{\mathrm{ho}}=$ $1 \mu \mathrm{m}$, and $\rho=10^{21} \mathrm{~m}^{-3}$, we get $t_{c} \simeq 10 / \omega_{\mathrm{tr}}$. For a harmonic trap with $\omega_{\mathrm{tr}}=100 \mathrm{~Hz}$, this amounts to $t_{c} \sim$ $10^{-1}$ seconds, clearly within the regime to be confirmed and studied experimentally [6].

\section{Lifetime for squeezed coherent groundstate}

The squeezed coherent state [12] $|\alpha, \gamma\rangle$ is defined as $|\alpha, \gamma\rangle=D(\alpha) S(\gamma)|v a c\rangle$ where

$$
S(\gamma)=\mathrm{e}^{\frac{\gamma}{2} a a-\frac{\gamma^{*}}{2} a^{\dagger} a^{\dagger}} .
$$

is the unitary squeezing operator and $D(\alpha)=\exp (\alpha a-$ $\left.\alpha^{*} a^{\dagger}\right)$ is the displacement operator. This again is a minimum uncertainty state, but the quantum fluctuations of two quadratures are not equal to each other. Arguments of $\gamma$ and $\alpha$ determine which quadrature is squeezed at the expense of increased uncertainty of the other one. In particular, if both parameters are real and positive, then the state is number squeezed, that is the uncertainty of the number operator is reduced whereas the conjugate variable, phase, has a higher uncertainty. Such a state resembles a Fock state more than a coherent state and therefore is expected to have a longer life time, since the phase collapse speed is generally proportional to $\Delta N$, which is smaller in this case, as have recently observed experimentally [14, 15]. This situation is analogous to the dispersion of a wavepacket consisting of different frequency components.

In terms of a new parameter

$$
\zeta=\gamma \frac{\tanh (|\gamma|)}{|\gamma|}
$$

the Fock state expansion of the squeezed coherent state is [16]

$$
\begin{aligned}
|\alpha, \gamma\rangle & =\sum_{n=0}^{\infty} A_{n}(\alpha, \zeta)|n\rangle \\
& \left.=\left(1-|\zeta|^{2}\right)^{1 / 4} \mathrm{e}^{-\frac{\left(\alpha+\zeta \alpha^{*}\right) \alpha^{*}}{2}} \sum_{n=0}^{\infty} \sqrt{\frac{\zeta^{n}}{2^{n} n !}} H_{n}\left(\frac{\alpha+\zeta \alpha^{*}}{\sqrt{2 \zeta}}\right) \mid(\dot{\theta})\right)
\end{aligned}
$$

Here, $H_{n}$ is the n-th Hermite Polynomial. The order parameter becomes

$$
\langle\alpha, \gamma|\hat{a}(t)| \alpha, \gamma\rangle=\sum_{n=0}^{\infty} \sqrt{n+1} A_{n}^{*} A_{n+1} \mathrm{e}^{\frac{i}{\hbar}\left(E_{n}-E_{n+1}\right) t},(10
$$

which is not possible to evaluate analytically. We therefore attack the problem using simple numerical methods, 
and plot the time dependence of the order parameter for various values of $\zeta$, both real and imaginary. It is clearly seen in figure1 1hat squeezing in the number direction increases the life time, whereas in figure 2 we see squeezing in phase direction leads to a faster decay.

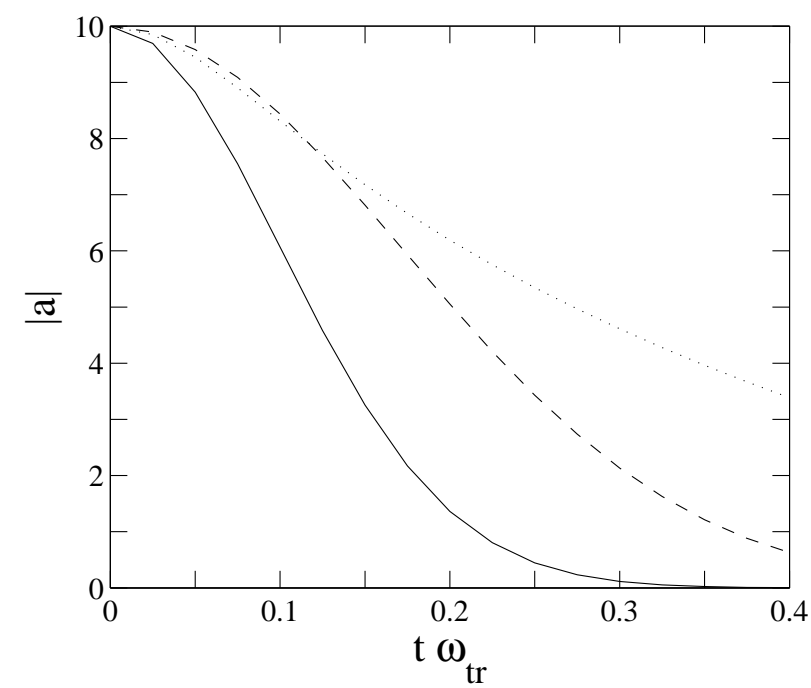

FIG. 1: The comparison of the short time decay character for a coherent state condensate with that for squeezed states with $\zeta=0.5$ and $\zeta=0.9$. Sample parameters used are $a_{s}=10 \mathrm{~nm}$, $a_{\mathrm{ho}}=1 \mu \mathrm{m}, n=10^{21} \mathrm{~m}^{-3}$, and $\alpha=10$ corresponds to $N=100$. In this case, the dimensionless time is in units of $\hbar / \tilde{u}$ becomes $\hbar / \tilde{u}=\omega_{\mathrm{tr}}^{-1}$. The fastest decay (solid line) is for the coherent state, while the dashed (dotted) line refers to that of a squeezed state with $\zeta=0.5(\zeta=0.9)$.

Considering the time evolution of the Q-functions might provide some extra insight into the phase diffusion. In a contour plot of a $\mathrm{Q}$ function, radial distribution corresponds to the number distribution whereas angular one gives the phase information. Beginning with a state with some phase information, we expect it to get a rotationally symmetric form as time passes and phase diffusion occurs. This is seen in the figures 3 and 4 which correspond to squeezed coherent states with $\alpha=10$ and $\zeta=\mp 0.5$ respectively.

\section{Lifetime for thermal coherent groundstate}

In order to study groundstate lifetime in finite temperature, we need a state with both a thermal characteristic and phase information. The thermal state with the density matrix

$$
\begin{aligned}
\rho_{\mathrm{th}} & =\mathrm{e}^{-\beta \mathcal{H}} \\
& =\sum_{n} \mathrm{e}^{-\beta E_{n}}|n\rangle\langle n|,
\end{aligned}
$$

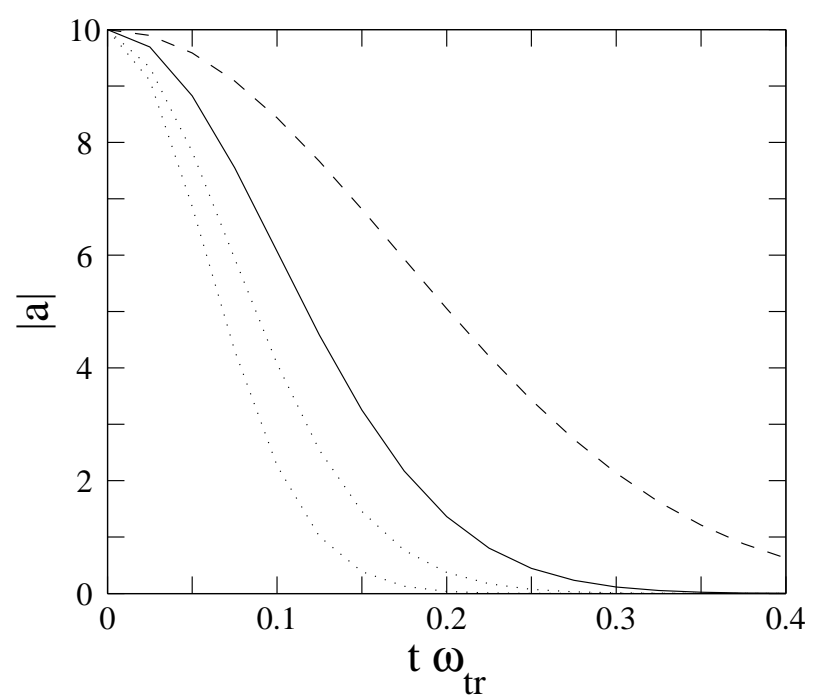

FIG. 2: Decay of the order parameter for the coherent state and squeezed states of $\zeta=0.5, \zeta=0.5 i$ and $\zeta=-0.5$ as a function of $t \omega_{t r}$. The same sample parameters with the previous plot are used. The solid line is the coherent state, the dashed line is the squeezed state with $\zeta=0.5$, and the dotted ones are the squeezed states with $\zeta=0.5 i$ and $\zeta=-0.5$.
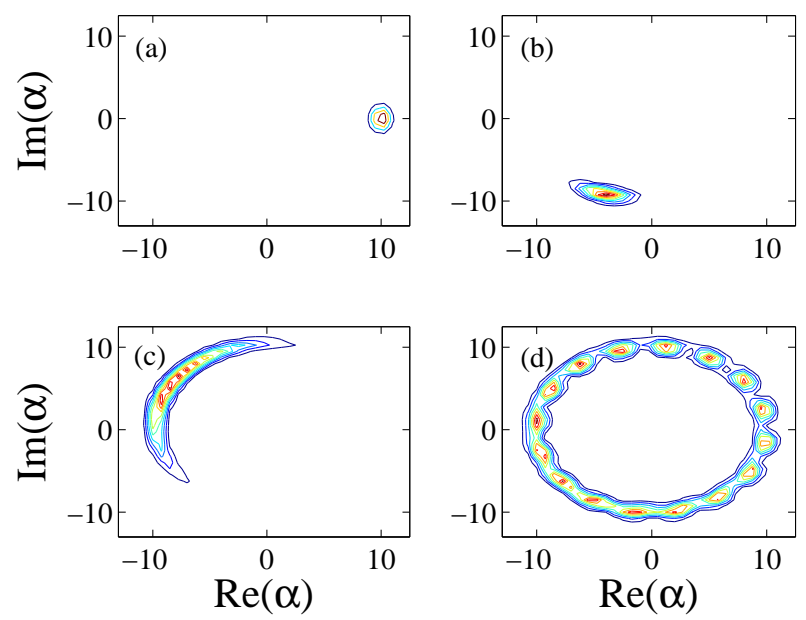

FIG. 3: Time evolution of the Q-function for squeezedcoherent state with $\alpha=10$ and $\zeta=0.5$ for increasing values of $t \omega_{t r}$. Figures (a), (b), (c) and (d) correspond to $t \omega_{t r}=0$, $t \omega_{t r}=0.02, t \omega_{t r}=0.10$ and $t \omega_{t r}=0.40$.

has a uniform phase, that is $\langle a\rangle=0$. We introduce the thermal coherent state as $\rho=D(\alpha) \rho_{\text {th }} D^{\dagger}(\alpha)$, where $\rho_{\text {th }}$ is defined using a Hamiltonian without free energy, that is $E_{n}|n\rangle=(\tilde{u} / 2) a^{\dagger} a^{\dagger} a a|n\rangle$. Using the Fock state expansion 

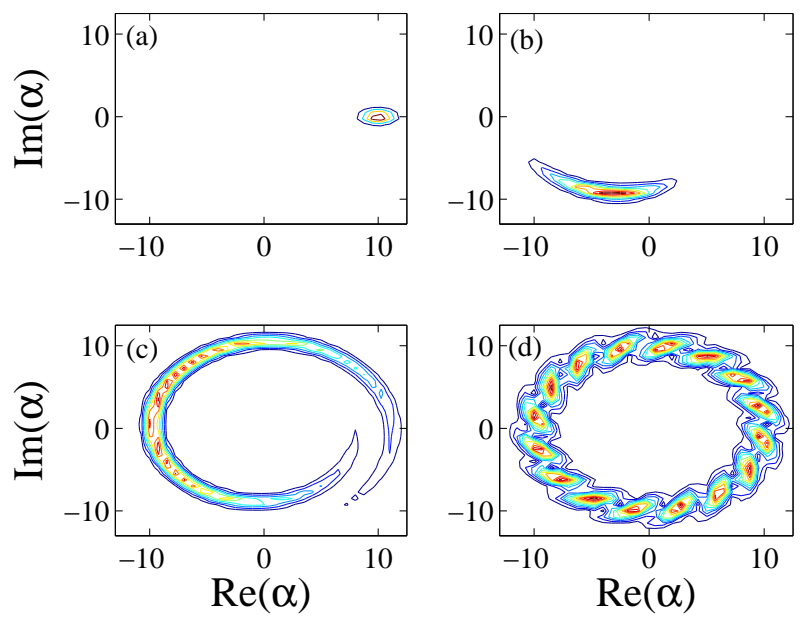

FIG. 4: Time evolution of the Q-function for squeezedcoherent state with $\alpha=10$ and $\zeta=-0.5$ for increasing values of $t \omega_{t r}$. Figures (a), (b), (c) and (d) again corresponds to same time steps; $t \omega_{t r}=0, t \omega_{t r}=0.02, t \omega_{t r}=0.10$ and $t \omega_{t r}=0.40$. The speed of phase diffusion is higher since the state is a phase-squeezed state.

of displaced number state $|n, \alpha\rangle[17]$

$$
\begin{aligned}
D(\alpha)|n\rangle & =|n, \alpha\rangle \\
& =\sum_{m=0}^{\infty} \mathrm{e}^{-\frac{1}{2}|\alpha|^{2}} \sqrt{\frac{n !}{m !}} \alpha^{m-n} L_{n}^{m-n}\left(|\alpha|^{2}\right)|m\rangle \\
& =\sum_{m=0}^{\infty} C_{m}(n, \alpha)|m\rangle,
\end{aligned}
$$

where $L_{k}^{l}$ are the generalized Laguerre polynomials, $\rho$ becomes

$$
\rho=\sum_{n m m^{\prime}} \mathrm{e}^{-\beta E_{n}} C_{m}(n, \alpha) C_{m^{\prime}}^{*}(n, \alpha)|m\rangle\left\langle m^{\prime}\right|
$$

The order parameter, which is found by taking the trace of $a \rho$ equals

$$
\begin{aligned}
\langle a(t)\rangle & =\sum_{n m m^{\prime} k} \mathrm{e}^{-\beta E_{n}} C_{m}(n, \alpha) C_{m^{\prime}}^{\dagger}(n, \alpha)\langle k \mid m\rangle\left\langle m^{\prime}\right| \mathrm{e}^{\frac{i}{\hbar} \mathcal{H} t} a \mathrm{e}^{-} \\
& =\sum_{n m} \mathrm{e}^{-\beta E_{n}} C_{m+1}(n, \alpha) C_{m}^{*}(n, \alpha) \sqrt{m} \mathrm{e}^{-\frac{i}{\hbar}\left(E_{m+1}-E_{m}\right) t}
\end{aligned}
$$

There will be destructive interference since $E_{n+1}-E_{n}$ is not constant. The number of contributing factors is determined by $\beta$, that is, temperature. Increased temperature will make more terms contribute and hence lead to shorter lifetime. Time evolution of the order parameter is plotted in figure 5 for $\alpha=10$.

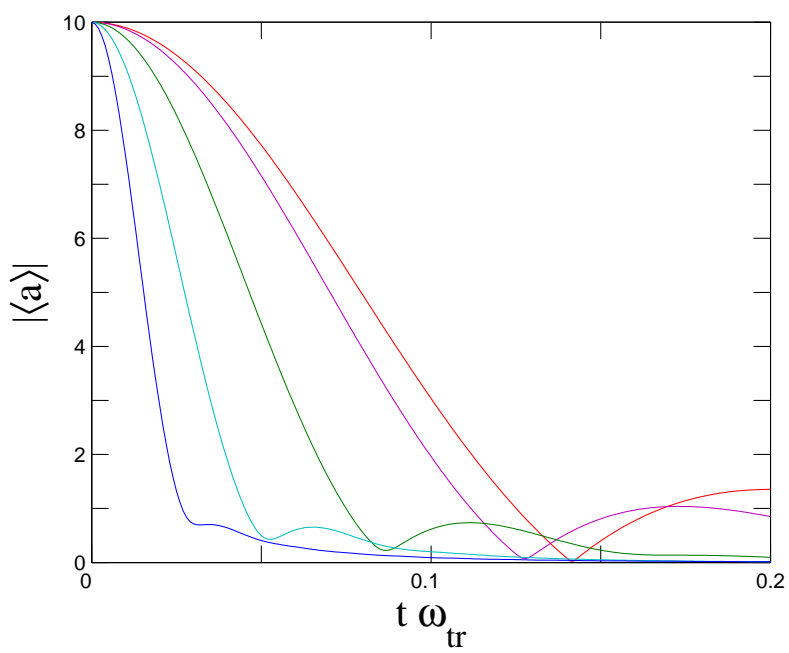

FIG. 5: The short time decays for thermal coherent states. The lines correspond respectively to $T=1000 \mathrm{nK}, 100 \mathrm{nK}$, $10 \mathrm{nK}, 1 \mathrm{nK}$, and $0.001 \mathrm{nK}$ from left to right. The humps are due to the ground degeneracy $E_{0}=E_{1}$. Even as the temperature approaches zero, the state does not approach the ordinary coherent state $D(\alpha)|0\rangle$. Instead, it approaches a superposition state $D(\alpha)(|0\rangle+|1\rangle) / \sqrt{2}$. It can be seen that the envelope of the function for small $T$ decays at the same time scale as a coherent state.

\section{E. Toy Model for Double Well Potential}

We now consider the case when two condensates in identical potential wells are brought into contact via a Josephson-like junction. The toy model Hamiltonian is of the form [10, 11]

$$
\mathcal{H}=\frac{\tilde{u}}{2}\left(a^{\dagger} a^{\dagger} a a+b^{\dagger} b^{\dagger} b b\right)-\mu\left(a^{\dagger} a+b^{\dagger} b\right)-\lambda\left(a^{\dagger} b+b^{\dagger} a\right)
$$

where $a(b)$ is the annihilation operator for the zero mode of the condensate in well A (B). We denote the state which has $n$ atoms in well $\mathrm{A}$ and $m$ atoms in well $\mathrm{B}$ by $|n, m\rangle$. Assuming the total number of atoms in both wells is fixed and equal to $N$, the ground state $|g r\rangle$ of the double well condensate can be expanded as

$$
|g r\rangle=\sum_{n=0}^{\infty} c_{n}|n, N-n\rangle .
$$

For identical wells, $c_{n}$ is expected to be peaked around $n=155) 2$. The strength $\lambda$ of the coupling determines the
dispersion of the number of atoms in a well. In particular, if one makes the ansatz

$$
\left|c_{n}\right|^{2} \propto \mathrm{e}^{-\frac{(n-N / 2)^{2}}{2 \sigma^{2}(N / 2)}}
$$

expanding the Schroedinger equation gives [10, 11]

$$
\sigma^{2}=\frac{N}{4} \sqrt{\frac{\lambda}{N \tilde{u} / 2+\lambda}} .
$$



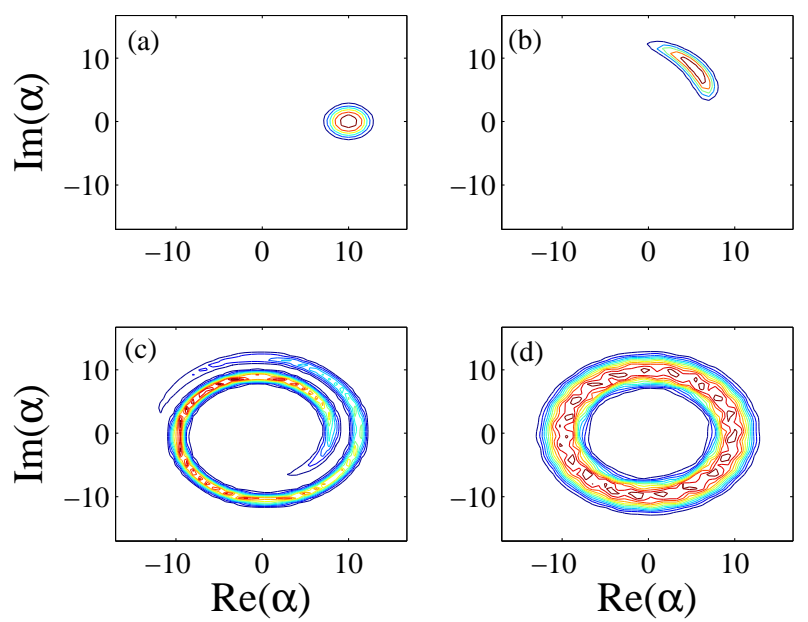

FIG. 6: Time evolution of the Q-function of a thermal coherent state with $\alpha=10$ at $T=10 \mathrm{nK}$. Figures (a), (b), (c) and (d) correspond to $t \omega_{t r}=0, t \omega_{t r}=10^{-4}, t=\omega_{t r}=10^{-3}$ and $t \omega_{t r}=10^{-2}$.
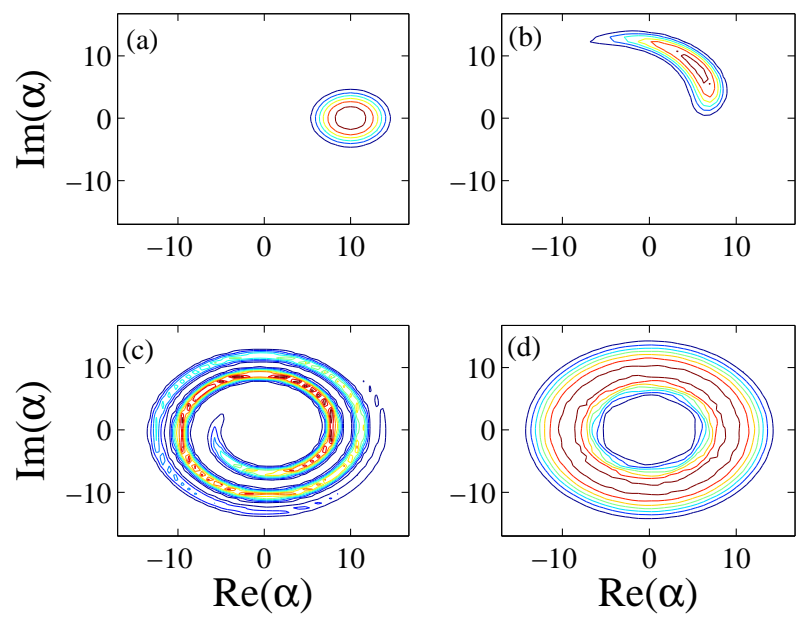

FIG. 7: Time evolution of the Q-function of a thermal coherent state with $\alpha=10$ at $T=100 \mathrm{nK}$. Figures (a), (b), (c) and (d) correspond to $t \omega_{t r}=0, t \omega_{t r}=10^{-4}, t \omega_{t r}=10^{-3}$ and $t \omega_{t r}=10^{-2}$. Wider radial distribution shows that even when the phase information lost, there is a higher uncertainty in number due to higher temperature.

In order to study the phase collapse, we consider the correlation $G=\left\langle g r\left|b^{\dagger} a\right| g r\right\rangle$. The time evolution of this expectation value after the coupling is turned off can be easily shown to be

$$
G=\sum_{n=0}^{\infty} \mathrm{e}^{-\frac{i}{\hbar}\left(E_{n}-E_{n+1}\right) t} c_{n}^{*} c_{n+1} \sqrt{(n+1)(N-n)} .
$$

We have defined $\mathcal{H}|n, N-n\rangle=E_{n}|n, N-n\rangle$, so $E_{n}=$
$\frac{\tilde{u}}{2}(n(n-1)+(N-n)(N-n-1))-\mu N$. Plotting this for coherent, number squeezed and phase squeezed states we see that the qualitative results we got from single well toy model is still valid, as expected.

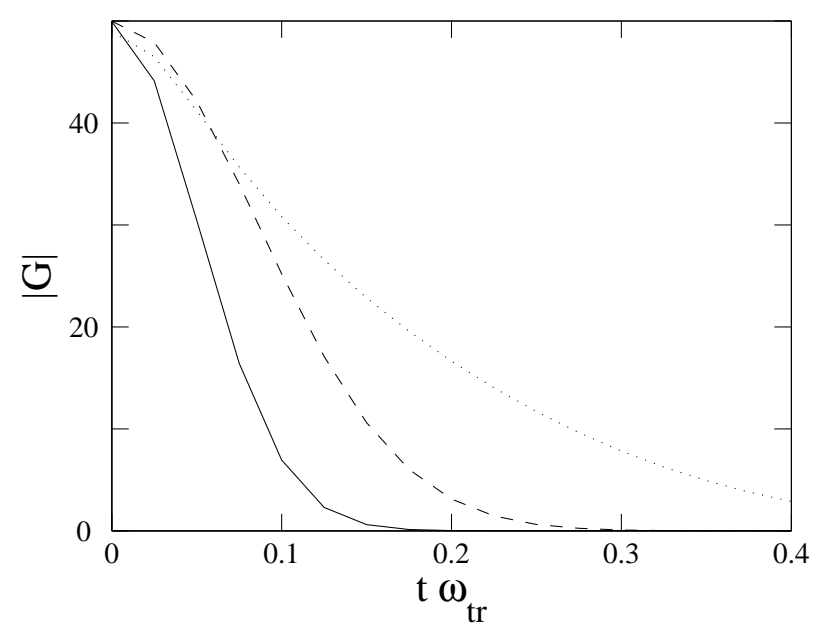

FIG. 8: Comparison of the short time decay for a double well condensate which has 200 atoms equally distributed to two wells. Solid line corresponds to a coherent state, dashed line corresponds to a squeezed state with $\zeta=0.5$ and dotted line to a squeezed state with $\zeta=0.9$.

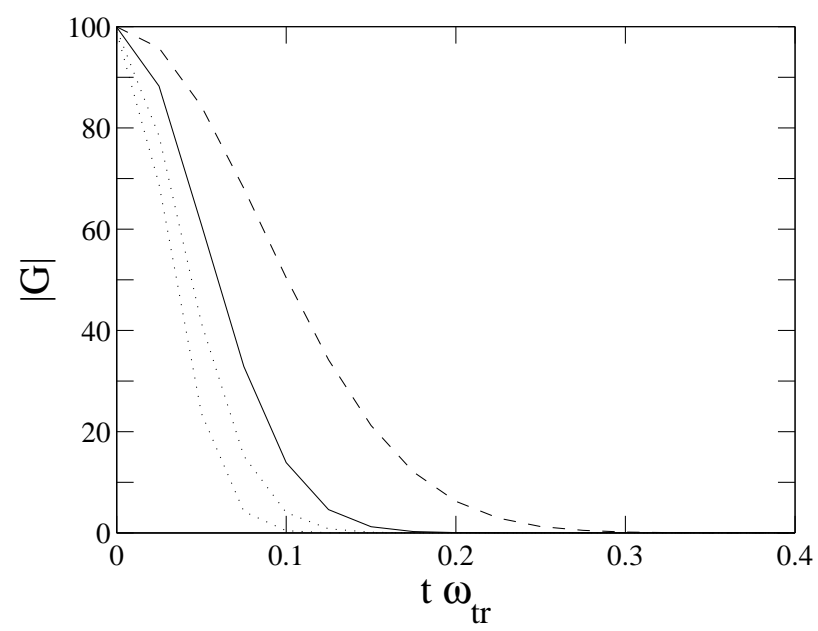

FIG. 9: Comparison of the short time decay for a double well condensate which has 200 atoms equally distributed to two wells. Solid line corresponds to a coherent state, dashed line corresponds to a squeezed state with $\zeta=0.9$ and dotted lines to squeezed states with $\zeta=0.5 i$ and $\zeta=-0.5$.

When the results for single and double well systems are compared, it is seen that although decay times are both in the same order of magnitude, a double well condensate with equal number of atoms in each well as a single 
well condensate suffers faster decay. This agrees with the analytical results obtained in [10] using the same ansatz for single and double well ground states.

\section{THIN SPECTRUM FORMALISM}

\section{A. Introduction}

By thin spectrum, we refer to a group of states whose energy spacings are so low that they are not controllable by any experiment and whose effect on the free energy becomes zero in the thermodynamic limit. That the existence of a thin spectrum leads to decoherence of excitations at finite temperature is proved in [7, 8]. In [8] it is shown that excitations on a Lieb-Mattis system suffer decoherence with a rate proportinal to $k_{B} T / N \hbar$ where $N$ is the system size. In [8] the same authors claim that this time scale, being independent of the details of the system, applies to other physical systems too, and in [9] they prove that Hubbard model superconductors suffers the same decay. In this section, we apply the thin spectrum formalism to atomic BECs and show that they suffer collapse in the same time scale.

\section{B. Quasiparticle lifetime in Bose-Einstein condensates}

We now go back to the Hamiltonian (1)

$$
\mathcal{H}=\sum_{k} E_{k} a_{k}^{\dagger} a_{k}+\frac{\tilde{u}}{2} \sum_{k, p, q} a_{p+q}^{\dagger} a_{k-q}^{\dagger} a_{k} a_{p} .
$$

Omitting the $3^{\text {rd }}$ and $4^{\text {th }}$ order terms in the noncondensed modes $(k \neq 0)$ we get

$$
\begin{gathered}
\mathcal{H}=\mathcal{H}_{z}+\mathcal{H}_{e}, \\
\mathcal{H}_{z}=\frac{\tilde{u}}{2}\left(\hat{n}_{0}^{2}-\hat{n}_{0}\right), \\
\mathcal{H}_{e}=\sum_{k \neq 0}\left[\left(E_{k}+2 \tilde{u} \hat{n}_{0}\right) \hat{n}_{k}+\frac{\tilde{u}}{2}\left(a_{k}^{\dagger} a_{-k}^{\dagger} a_{0} a_{0}+\text { h.c. }\right)\right] .
\end{gathered}
$$

In order to study the excitations and zero mode separately, we need $\left[\mathcal{H}_{z}, \mathcal{H}_{e}\right]=0$ and for this we neglect the quantum nature of $a_{0}$ in $\mathcal{H}_{e}$ by replacing $\hat{n}_{0} / V$ appearing in $\mathcal{H}_{e}$ by $\rho_{0}=N_{0} / V$. Here $N_{0}$ denotes the number of atoms in the zero mode, and so $\rho_{0}$ is the corresponding density. After substituting the chemical potential that gives the correct number of atoms, $\mu_{0}=u_{0} \rho_{0}-u_{0} \rho_{0} / 2 N_{0}$, we get

$$
\mathcal{H}=\frac{u_{0} \rho_{0}}{2 N_{0}} \hat{n}_{0}^{2}-\rho_{0} u_{0} \hat{n}_{0}+\mathcal{H}_{e}
$$

$$
\mathcal{H}_{e}=\sum_{k \neq 0}\left[\epsilon_{k} \hat{n}_{k}+\frac{u_{0} \rho_{0}}{2}\left(a_{k}^{\dagger} a_{-k}^{\dagger}+\text { h.c. }\right)\right] .
$$

Here, $u_{0}$ is the interaction strength not scaled with $\mathrm{V}$, that is $u_{0}=V \tilde{u}$, and $\epsilon_{k}$ is defined as $\epsilon_{k}=E_{k}+2 u_{0} \rho_{0}$. The excitation Hamiltonian can be diagonalized to give

$$
\mathcal{H}_{e}=\sum_{k \neq 0} \omega_{k} b_{k}^{\dagger} b_{k}+\text { const. }
$$

with $\omega_{k}=\left[\epsilon_{k}^{2}-u_{0}^{2} \rho_{0}^{2}\right]^{1 / 2}[18]$ and $b_{k}=S a_{k} S^{-1} . S$ is the multi-mode squeeze operator [19].

For simplicity, we consider a system with quasiparticle excitations in only one mode. We denote such a system with $n$ atoms in the condensate and $m$ quasiparticles with $\omega$ by $|n, m\rangle$. Then,

$$
\begin{aligned}
\hat{n}_{0}|n, m\rangle & =n|n, m\rangle, \\
\hat{n}_{k^{\prime}}|n, m\rangle & =m \delta_{k, k^{\prime}}|n, m\rangle, \\
\mathcal{H}|n, m\rangle & =E_{m}^{(n)}|n, m\rangle,
\end{aligned}
$$

We consider single-particle regime such that quasiparticle and particle occupation numbers become the same. Due to the number conservation, sum of condensate atoms and the quasiparticles should remain the same. To excite $m$ quasiparticles, we have to decrease condensate atom number by $m$. The energy of the corresponding state becomes

$$
E_{m}^{(n)}=\left[\frac{u_{0} \rho_{0} n^{2}}{2\left(N_{0}-m\right)}-u_{0} \rho_{0} n+m \omega\right] .
$$

Following [7], we assume that in the beginning the system has no quasiparticle excitations at all, and therefore has a Boltzmann weighted distribution over the states $|n, 0\rangle$, i.e.,

$$
\rho(t=0) \propto \sum_{n} \mathrm{e}^{-\beta E_{0}^{(n)}}|n, 0\rangle\langle n, 0| .
$$

This state has no phase, and therefore is not the perfect starting point for a BEC. However, if we are interested only in the collapse of the excitations and if this take place on a time scale smaller than the time of phase diffusion of the zero mode, then we can get an estimate. For the time being we will study excitations on this thermal state, and in the following subsection we will generalize our ideas to thermal coherent states.

Before proceeding further, we replace the sum in eq. (32) by an integral. Since the value of $E_{n}$ will be extremely small for $n<0$, it is also legitimate to expand this integral to include the negative values of $n$ too.

Now we bring the system to a superposition of the zero-quasiparticle state and the one quasiparticle state, that is we bring each $|n, 0\rangle$ to $(|n, 0\rangle+|n, 1\rangle) / \sqrt{2}$. Such a state can be interpreted as a particular qubit [7]. For thin spectrum to affect the system, it is essential that we bring the system to a superposition rather than simply 
exciting a quasiparticle. Now, the off diagonal element of the resulting state's density matrix will evolve according to

$$
\begin{aligned}
\rho_{\text {od }}(t>0) & \propto \int_{-\infty}^{\infty} \mathrm{e}^{-\beta E_{0}^{(n)}} \mathrm{e}^{-\frac{i}{\hbar}\left(E_{1}^{(n)}-E_{0}^{(n)}\right) t} d n \\
& \propto \int_{-\infty}^{\infty} \mathrm{e}^{\left(-\beta u_{0} \rho_{0} / 2 N_{0}+i t u_{0} \rho_{0} / 2 \hbar N_{0}^{2}\right) n^{2}+\beta \rho_{0} u_{0} n} d n \\
& \propto \sqrt{\pi} \frac{\exp \left(\frac{\beta^{2} \rho_{0}^{2} u_{0}^{2}}{2 \beta u_{0} \rho_{0} / N_{0}-2 i t u_{0} \rho_{0} / \hbar N_{0}^{2}}\right)}{\sqrt{\beta u_{0} \rho_{0} / 2 N_{0}-i t u_{0} \rho_{0} / 2 \hbar N_{0}^{2}}},
\end{aligned}
$$

which gives

$$
\left|\rho_{\text {od }}(t)\right|^{2} \propto \frac{\exp \left(\frac{\beta^{3} N_{0}^{3} u_{0} \rho_{0}}{\beta^{2} N_{0}^{2}+t^{2} / \hbar^{2}}\right)}{\sqrt{\beta^{2}+t^{2} / \hbar^{2} N_{0}^{2}}},
$$

after omitting terms with only a phase factor. Although the denominator and the numerator have quite different forms, we find that both decay in a time proportional to $t_{c} \sim \hbar N_{0} / k_{B} T$. This is the same result that Wezel et. al. have found for a crystal [7].

For a BEC, we can let $N_{0} \sim 10^{6}-10^{8}$ and $T \sim$ $10^{-8}-10^{-7} \mathrm{~K}$. This gives $t_{c} \sim 10^{2}-10^{5}$ seconds, which is much larger than even the ground state life times. Unlike the room temperature mesoscopic system discussed in [7], BECs are extremely cold systems therefore one single excitation has such a long life time. However, this does not make the calculation unuseful. This life time is given only for a single quasi-particle excitation. In general it might be useful to have more than one quasiparticles excited at a time. If, for example, the condensate will be used as the building block of a quantum computer, having an excitation consisting of $\sim N$ quasiparticles will make observation of the qubit (superpositions of states with $m=0$ and $m$ quasiparticles), easier. In order to find a decay time for $m>1$, the only approximation required is $1 / N(N-m) \simeq 1 / N^{2}$ and the timescale will be inversely proportional to $\mathrm{m}$. For $m \sim N_{0}$, so long as it is not the case that $1-m / N \ll 1$, we have $t_{c} \sim 10^{-4}-10^{-3}$ seconds. This time scale is much smaller than both the observed and expected ground state life times, therefore is of interest.

There are different studies [20, 21] concerning the life times of quasiparticle excitations, such as using perturbation theory, etc. Namely, [20] has found a linear temperature dependence for high energy quasiparticles. Our calculation will make a quantitative contribution to this decay rate. However, low energy excitations are shown to have more complex temperature dependencies [21]. Our calculations do not make any predictions for that regime, since we have assumed $E_{k} \gg u_{0} \rho_{0}$. Also, our theory predicts a certain dependence of the life time on the number of quasiparticles excited (which is almost linear for small $m$ ), and this might be used to differentiate it from other theories.

As discussed before, for a group of states to be a thin spectrum, their effect on the free energy must vanish. For this purpose, we write the partition function as

$$
Z=Z_{\text {thin }} \cdot Z_{\text {observable }}
$$

with $Z_{\text {thin }}=\sum_{n} \mathrm{e}^{-\beta u_{0} \rho_{0}\left(n^{2} / 2 N_{0}-n\right)}$. Again replacing the sum by an integral we find that the leading term in the free energy per particle $\ln \left(Z_{\text {thin }}\right) / N_{0} \sim \ln \left(N_{0}\right) / N_{0}$. This means that the mode we consider has no effect on free energy and satisfies all the criteria to constitute a thin spectrum.

The next step might be to generalize the calculations for coherent of squeezed coherent zero mode occupations. However, following this path doesn't give any finite life time, since the excitation decay due to thin spectrum requires finite temperature, but coherent states have no temperature characteristic. But it is natural to do the same calculations for thermal coherent zero mode occupation. Such a calculation is presented in [1]. Decay rate does not have a linear temperature dependence in this case, therefore it might be possible to differentiate between thermal and thermal coherent occupations experimentally.

\section{Effect of Thin Spectra on a Double Well Condensate}

In [10], it is shown that the phase related part of the Hamiltonian of a double well condensate can be reduced to the form

$$
\mathcal{H}=\alpha_{+} P_{+}^{2}+\alpha_{-} P_{-}^{2}+\lambda \gamma_{-} Q_{-}^{2} .
$$

Here, $\alpha_{+}, \alpha_{-}$and $\gamma_{-}$are parameters depending on system details, $P_{+}$and $P_{-}$are the momenta corresponding to the total and relative phases of the condensates, and $Q_{-}$is the coordinate corresponding to the relative phase. $\lambda$, again, is a number parameterizing the tunneling between different wells. (This Hamiltonian, originally derived for a condensate consisting of two different types of atoms in a single potential well, is applicable to a double well system when the parameter corresponding to collisions between different types of atoms is taken to be zero.) When coupling $\lambda$ between the wells is taken to be zero, this Hamiltonian reduces to one of two free particles:

$$
\mathcal{H}=\alpha_{+} P_{+}^{2}+\alpha_{-} P_{-}^{2} .
$$

It is seen that the system has two modes corresponding to motions without restoring forces. The reason is that now that there are two wells, there is an extra symmetry that is spontaneously broken. Therefore, there are two different thin spectra.

Cumulative effect of multiple broken symmetries (and hence multiple thin spectra) on excitation lifetime is studied in [1]. If lifetime corresponding to individual thin spectra are $t_{1}$ and $t_{2}$, then the resultant collapse time is the harmonic sum of individual life times:

$$
t_{r}^{-1}=t_{1}^{-1}+t_{2}^{-1} \text {. }
$$


Using $\alpha$ 's corresponding to the system under consideration, it is thus possible to find the resultant life time, which is supposed to be in the same order of magnitude with the smaller life time.

\section{CONCLUSIONS}

Generalizing the Toy model calculations [10, 11], we discussed the phase decoherence of coherent, squeezed coherent and thermal coherent states. For visual clarity, time dependence of various $\mathrm{Q}$ functions is shown. A generalization of the toy model to double well systems is also discussed and time evolution of the order parameter is studied for coherent and squeezed coherent states. This step, being important not only for double well BECs, might bear important results for any Josephson-coupled system.
The effect of thin spectrum [7, 8] on quasiparticle excitations in BECs is briefly reviewed. It is shown that the presence of the so called thin spectrum states, which have vanishing level spacing, also has no effect on free energy per particle in the thermodynamic limit. Qualitative dependence of life time on the number of excitations is given. Finally, as a simple example of a system with more than one spontaneously broken symmetry [1], a calculation of excitation life time in a double well system is outlined.

\section{ACKNOWLEDGEMENTS}

T.B. is supported by TÜBİTAK. O.E.M. acknowledges the support from a TÜBA/GEBIP grant. T.B. acknowledges fruitful discussions with Jasper van Wezel and Patrick Navez.
[1] T. Birol et al., Phys. Rev. A 76, 043616 (2007).

[2] S. N. Bose, Z. Phys. 26, 178 (1924); A. Einstein, Sitzungsber. K. Preuss. Akad. Wiss. 22, 261 (1924); ibid 23, 3 (1925).

[3] M. H. Anderson et. al., Science 269, 198 (1995); C. C. Bradley et. al., Phys. Rev. Lett. 75, 1687 (1995); K. B. Davis et. al., Phys. Rev. Lett., 75, 3969 (1995).

[4] E.M. Wright, D.F. Walls, and J.C. Garrison, Phys. Rev. Lett. 77, 2158 (1996).

[5] M. Lewenstein and L. You, Phys. Rev. Lett. 77, 3489 (1996).

[6] M. Greiner, O. Mandel, T. W. Hansch, and I. Bloch, Nature 419, 51 (2002).

[7] J. van Wezel, J. Zaanen, and J. van den Brink, Phys. Rev. B 74, 094430 (2006).

[8] J. van Wezel, J. van den Brink, and J. Zaanen, Phys. Rev. Lett. 94230401 (2005).

[9] J. van Wezel, J. van den Brink, arxiv:cond-mat/07043703 (2007).

[10] P. Villain et. al, J. of Mod. Optics 44, 1775 (1997).

[11] A. Imamoglu, M. Lewenstein, and L. You, Phys. Rev. Lett. 78, 2511 (1997).
[12] L. Mandel and E. Wolf, Optical Coherence and Quantum Optics, Cambridge University Press (1995), page 1038.

[13] C. Cohen Tannoudji, B. Diu, F. Laloe, Quantum $\mathrm{Me-}$ chanics, Wiley \& Sons (1977).

[14] G.-B. Jo, Y. Shin, S. Will, T. A. Pasquini, M. Saba, W. Ketterle, D. E. Pritchard, M. Vengalattore, and M. Prentiss, Phy. Rev. Lett. 98, 030407 (2007).

[15] F. Gerbier, S. Foelling, A. Widera, O. Mandel, and I. Bloch, Phys. Rev. Lett. 96, 090401 (2006).

[16] A. Wünsche in Theory of Nonclassical States of Light, edited by V. V. Dodonov and V. I. Man'ko, (Taylor and Francis, New York, 2003).

[17] S. M. Roy and C. Singh, Phys. Rev. D 25, 3413 (1982).

[18] W. Greiner, Quantum Mechanics-Special Chapters, (Springer-Verlag, New York, 1998), Chapter 6.

[19] M. Haque and A. E. Ruckenstein, Phys. Rev. A 74, 043622 (2006); P. Navez, Mod. Phys. Lett. B 12, 705 (1998).

[20] P. O. Fedichev and G. V. Shlyapnikov, Phys. Rev. A 58, 3146 (1998).

[21] W. V. Liu, Phys. Rev. Lett. 79, 4056 (1997). 